\title{
Évaluation de la teneur en vitamine A et de l'indice de peroxyde des huiles végétales couramment vendues dans les marchés au Burkina Faso
}

\author{
Tebkieta Marcelline OUEDRAOGO KANGAMBEGA ${ }^{1 *}$, Fulbert NIKIEMA $^{1}$, \\ Dissinviel Stéphane KPODA ${ }^{3}$, Moumouni BANDE ${ }^{1,2}$, Sandaogo SAWADOGO ${ }^{1}$, \\ Ouambila Isidore SAMA ${ }^{1}$, Inoussa ILBOUDO ${ }^{1}$, Bazoin Sylvain Raoul BAZIE ${ }^{1}$, \\ Muller Kiswendsida Abdou COMPAORE ${ }^{1}$, Naamwin-So-Bawfu Romaric MEDA ${ }^{1}$, \\ Asseto BELEMLOUGRI SOMDA ${ }^{1}$, Alphonse YAKORO ${ }^{1}$, Bernadette Pane OUATTARA \\ SOURABIE $^{1}$, Naa-Imwine Stanislas Dimitri MEDA ${ }^{1}$, Christian Sibiri KABORE ${ }^{1}$, \\ Hamado KARENE ${ }^{1}$, Hervé HIEN $^{4}$ et Elie KABRE ${ }^{1,2}$ \\ ${ }^{1}$ Laboratoire National de Santé Publique, 09 BP 24 Ouagadougou 09, Burkina Faso. \\ ${ }^{2}$ Université Joseph Ki-Zerbo, Unité de Formation et de Recherche en Sciences de la Santé, \\ 03 BP 7021 Ouagadougou 03, Burkina Faso. \\ ${ }^{3}$ Université Joseph Ki-Zerbo, Centre Universitaire de Ziniaré, 03 BP 7021 Ouagadougou 03, Burkina Faso. \\ ${ }^{4}$ Institut National de Santé Publique, BP 10278 Ouagadougou, Burkina Faso. \\ *Auteur correspondant; E-mail: marcelline_ok@yahoo.fr; Tél.: (00226) 78217111
}

\section{REMERCIEMENTS}

Ce travail a été réalisé dans le cadre du projet PAASME-UE ("Production et Analyses des données pour Améliorer la Santé de la Mère et de l'Enfant"). Ce projet de recherche est conjointement mis en ouvre par l'INSP et le LNSP. Il a été entièrement financé par l'Union Européenne (UE) qu'il convient donc de remercier au terme de ce travail.

\begin{tabular}{lll}
\hline Received: 10-08-2021 & Accepted: 23-12-2021 & Published: 31-12-2021 \\
\hline
\end{tabular}

\section{RÉSUMÉ}

La carence en vitamine A constitue un problème de santé publique dans les pays en développement et affecte en particulier les jeunes enfants et les femmes en âge de procréer. Le but de cette étude était d'évaluer l'application de la politique nationale en matière de production et d'importation, des huiles végétales raffinées, d'enrichissement obligatoire en vitamine A au Burkina Faso, à travers la détermination de la teneur en vitamine A et de l'indice de peroxyde des huiles végétales vendues sur le marché. Cette étude transversale a été menée dans cinq villes sélectionnées selon la densité de la population. Cinquante-neuf échantillons d'huile ont été achetés dans ces villes pour évaluer la teneur en vitamine A, l'indice de peroxyde et leur conformité aux normes nationales et internationales. Dans l'ensemble, 76,27\% des huiles n'étaient pas suffisamment enrichies en vitamine A. Pour l'indice de peroxyde, 3,39\% des huiles n'étaient pas conformes. Globalement, 77,97\% des huiles n'étaient pas conformes pour ces deux paramètres. Au regard de ces résultats il est nécessaire d'intensifier la lutte contre la mise à la consommation des huiles non enrichies en vitamine A au Burkina Faso.

(C) 2021 International Formulae Group. All rights reserved.

Mots clés : Vitamine A, huiles végétales, indice de peroxyde, Burkina Faso. 


\title{
Evaluation of the vitamin A content and the peroxide value of vegetable oils commonly sold in markets in Burkina Faso
}

\begin{abstract}
Vitamin A deficiency is a public health problem in developing countries and particularly affects young children and women of childbearing age. The aim of this study was to assess the implementation of the national policy on the production and importation of refined vegetable oils and mandatory vitamin A fortification in Burkina Faso, through the determination of the vitamin A content and peroxide value of vegetable oils sold on the market. A cross-sectional study was conducted in five cities selected according to population density. Fifty-nine oil samples were purchased in these cities to assess vitamin A content, peroxide value and compliance with national and Codex standards. Overall, $76.17 \%$ of the oils were not adequately fortified with vitamin A. For the peroxide value, $3.39 \%$ of the oils did not comply. Overall, $77.97 \%$ of the oils did not comply for these two parameters. In view of these results, it is necessary to intensify the fight against the consumption of oils not enriched in vitamin A in Burkina Faso.
\end{abstract}

(C) 2021 International Formulae Group. All rights reserved.

Keywords: Vitamin A, vegetable oils, peroxide value, Burkina Faso.

\section{INTRODUCTION}

La malnutrition par carence en micronutriments touche plus de deux milliards de personnes dans le monde, notamment les carences en vitamine $\mathrm{A}$, en iode, en fer et en zinc (FAO/WHO, 2015). Elle constitue un problème de santé publique dans les pays en développement. Les jeunes enfants et les femmes en âge de procréer constituent les groupes vulnérables en raison de l'augmentation de leurs besoins physiologiques.

La carence en vitamine A est la cause majeure de troubles visuels graves (xérophtalmie) et de cécité évitable chez l'enfant. La carence, même subclinique, a des répercussions sur la survie des enfants et la santé de leur mère (Mohamed Ag Bendech et al., 2000). La carence en vitamine A augmente également le risque de carence en fer et d'anémie, notamment par un effet négatif sur la mobilisation des réserves en fer (Hoag et al., 2002 ;Cusick et al., 2005; Schroeder et al., 2007 ; Citelli et al., 2012).

La carence en vitamine A résulte d'une faible consommation d'aliments riches en rétinol ou en caroténoïdes en particulier pendant certaines circonstances de la vie (période de croissance accélérée et au cours de la grossesse) (OMS/FAO, 2011).

Le Burkina Faso s'est engagé avec les pays de l'UEMOA dans le processus d'enrichissement de l'huile alimentaire en vitamine $\mathrm{A}$; et l'arrêté interministériel, N²012-0232/MICA/MS/MEF/MAH portant enrichissement obligatoire des huiles végétales raffinées en vitamine $\mathrm{A}$, interdit l'importation des huiles non enrichies en vitamine A sauf pour le cas des huiles diététiques (Ministère de la Santé, 2012). La promotion des aliments enrichis en vitamine A constitue une des interventions de la deuxième orientation stratégique du Plan stratégique multisectoriel de nutrition 2020-2021 (Ministère de la Santé, 2020). La supplémentation en vitamine A seule peut réduire le risque d'anémie en améliorant les taux d'hémoglobine et de ferritine chez les personnes dont le taux de rétinol sérique est faible (Marcela et al., 2018).

Les huiles végétales sont constituées à 99\% de triglycérides (esters glycériques et d'acides gras saturés, mono-insaturés et polyinsaturés), le reste étant composé principalement de lectines et de vitamine $\mathrm{E}$ ou 
tocophérol ayant des propriétés antioxydantes. Une forte concentration d'Acides Gras PolyInsaturés (AGPI) rend l'huile végétale plus sujette à l'oxydation pendant le stockage (Dubois et al., 2007). L'oxydation a lieu plus vite sous l'effet des rayons ultraviolets, de la chaleur ou en présence d'ions métalliques comme le $\mathrm{Fe}^{2+}$ ou le $\mathrm{Cu}^{2+}$. Elle modifie le goût et des composés indésirables (acides gras libres et peroxydes, radicaux libres) apparaissent entrainant une perte de la valeur nutritive de l'huile (Tao, 2015 ; Youma et al., 2015). Ces substances indésirables peuvent être dangereuses pour la santé, car elles peuvent induire un stress oxydatif et une inflammation lorsqu'elles sont à des concentrations élevées (Tao, 2015).

L'indice de peroxyde est un paramètre qui permet de mesurer la quantité d'oxygène chimiquement lié à une huile ou à corps gras sous forme de peroxydes, en particulier d'hydroperoxydes.

Le but de cette étude était d'évaluer l'application de la politique nationale relative à l'enrichissement obligatoire des huiles végétales raffinées en vitamine $\mathrm{A}$ à travers la détermination de la teneur en vitamine $\mathrm{A}$, et de l'indice de peroxyde des huiles végétales vendues au Burkina Faso.

\section{MATÉRIEL ET MÉTHODES}

\section{Type d'étude}

Il s'agissait d'une étude quantitative transversale qui a été réalisée de Décembre 2020 à Janvier 2021.

\section{Population d'étude / Échantillonnage / taille de l'échantillon et collecte des données}

L'étude a concerné cinq (5) des treize (13) régions administratives du Burkina Faso. Le choix de ces régions a consisté à organiser un sondage en grappes à deux degrés. Ainsi, dans le premier degré de stratification, cinq (5) régions ont été sélectionnées sur la base de la densité de la population et le trafic commercial au niveau des frontières. Il s'agit des régions du Centre, du Centre-Sud, du Centre-Est, des Cascades et des Haut Bassins. (Figure 1).
Une sous stratification a été réalisée dans chacune de ces régions pour choisir des marchés et boutiques pour la collecte des huiles. Ces marchés, boutiques et restaurants ont été tirés au sort dans les différentes villes sélectionnées pour l'étude.

L'échantillonnage a été réalisé sur la base d'une formule statistique. La taille de l'échantillon a été calculée selon la probabilité (p) qu'un produit acheté soit propre à la consommation. Le nombre d'échantillons d'huile à prélever a été déterminé en fonction de la densité de la population pour les villes de Ouagadougou et Bobo-Dioulasso et en fonction $\mathrm{du}$ trafic au niveau des postes frontaliers. Ainsi, dix-huit (18), douze (12), dix (10), dix (10) et neuf (09) échantillons d'huiles ont été collectés respectivement à Ouagadougou, Bobo-Dioulasso, Cinkansé, Niangoloko et Dakola.

A l'intérieur de chaque marché, les sites de prélèvement ont été sélectionnés pour l'achat des huiles selon la méthode des itinéraires. Au total, cinquante-neuf (59) échantillons d'huile ont été collectés et acheminés au Laboratoire national de santé publique dans les conditions requises.

\section{Méthodes d'analyse \\ Détermination de la teneur en vitamine A}

La méthode utilisée était une méthode adaptée de celle du dosage de la vitamine A dans l'huile de table par Chromatographie Liquide Haute Performance (CLHP de marque Thermo Hionex Ultimate couplé à un détecteur UV) selon la norme française NF T 90-210 Mai 2009 (Ait Errayess et al., 2014). Environ 2 g d'huile ont été dissous dans $20 \mathrm{~mL}$ d'acétone, la solution a été homogénéisée à l'aide d'un vortex, et injectée dans un vial à l'aide d'une seringue munie d'un filtre. La phase mobile était constituée de méthanol et la colonne était une C18 de type Zorbax. La longueur d'onde de détection était de $325 \mathrm{~nm}$. Le standard utilisé était le palmitate de rétinol (Rentyl palmitate : type IV, -1,800,000 USP unit/g, oil ; lot \# MKBR6526V). 
La teneur en équivalent rétinol a été calculée en utilisant la formule suivante :

Ce x Vd

$\operatorname{TvitA}(\mathrm{mg} / \mathrm{kg})=$

PE

Où

TvitA : représente la teneur en vitamine A,

$C e$ : représente la concentration lue par le chromatographe,

$V d$ : représente le volume de dissolution,

$P E$ : représente la prise d'essai,

Le résultat a été exprimé en $\mathrm{mg} / \mathrm{kg}$ d'huile.

\section{Détermination de l'indice de peroxyde}

L'indice de peroxyde a été déterminé par titrage iodométrique (ISO 3960 -1977 (F)). La méthode est basée sur le traitement d'une prise d'essai, en solution dans de l'acide acétique et du chloroforme, par une solution d'iodure de potassium. L'iode libéré a été titré à l'aide d'une solution de thiosulfate de sodium à $0,1 \mathrm{~N}$. Brièvement, environ $2 \mathrm{~g}$ d'huile ont été dissous dans $10 \mathrm{~mL}$ de chloroforme. Après addition de $15 \mathrm{~mL}$ d'acide acétique et $1 \mathrm{~mL}$ d'iodure de potassium saturé, la solution a été agitée pendant $1 \mathrm{~min}$ et placée pendant $5 \mathrm{~min}$ dans le noir. Après addition de $75 \mathrm{~mL}$ d'eau distillée et quelques gouttes d'indicateur d'amidon (1\%), la solution a été titrée avec du thiosulfate de sodium $0,1 \mathrm{~N}$ jusqu'à ce que la couleur bleue disparaisse. Un blanc, constitué par le milieu réactionnel sans l'analyte a été préparé suivant la même procédure que les échantillons et puis titré. L'indice de peroxyde de chaque échantillon d'huile a été calculé en utilisant la formule suivante :

$$
\operatorname{IP}(\mathrm{mEq} / \mathrm{kg})=\frac{\left(\mathrm{V}_{1}-\mathrm{V}_{\mathrm{o}}\right) \times \mathrm{T}}{\mathrm{m}} \times 1000
$$

Où

$V_{o}$ : représente le volume, en millilitres, de la solution titrée de thiosulfate de sodium, utilisé pour l'essai à blanc ;
$V_{l}$ : représente le volume, en millilitres, de la solution titrée de thiosulfate de sodium, utilisé pour la détermination;

T: représente la normalité de la solution de thiosulfate de sodium utilisée ;

$m$ : représente la masse, en grammes, de la prise d'essai.

Chaque échantillon a été analysé en duplicat essais et le résultat par échantillon a été calculé en faisant la moyenne arithmétique des deux déterminations effectuées. Selon la teneur en indice de peroxyde obtenue, la différence entre les résultats de deux déterminations, effectuées simultanément ou rapidement l'une après l'autre, par le même analyste, sur le même échantillon, ne doit pas dépasser les valeurs inscrites au Tableau 1.

\section{Conformité des paramètres analysés aux normes de spécification}

La norme pour les huiles végétales CXS 210-1999, révisée en 2019; a été utilisée pour apprécier la conformité des indices de peroxyde des huiles analysées au cours de cette étude. Selon cette norme, la limite maximale tolérée de l'indice de peroxyde des huiles raffinées est 10 milliéquivalents d'oxygène actif par kilogramme d'huile.

Les normes sur les huiles comestibles raffinées enrichies en vitamine A (NBF 01187:2010 - huile de palme ; NBF 01-188:2010 - huile de coton ; NBF 01-189:2010 - huile d'arachide ; NBF 01-190:2010 - huile de soja ; NBF 01-191:2010 - huile comestible de palmiste ; NBF 01-192:2010- huile comestible de tournesol raffinée ; NBF 01-194:2010 huile de colza ; NBF 01-195:2010 - huile de maiis) ont été utilisées pour apprécier la conformité des teneurs en vitamine A des huiles prélevées au cours de cette étude.

\section{Traitement statistique des données}

Les résultats d'analyses de laboratoire ont été saisis sur le tableur Excel et traités à l'aide du logiciel GraphPad Prism 5. 


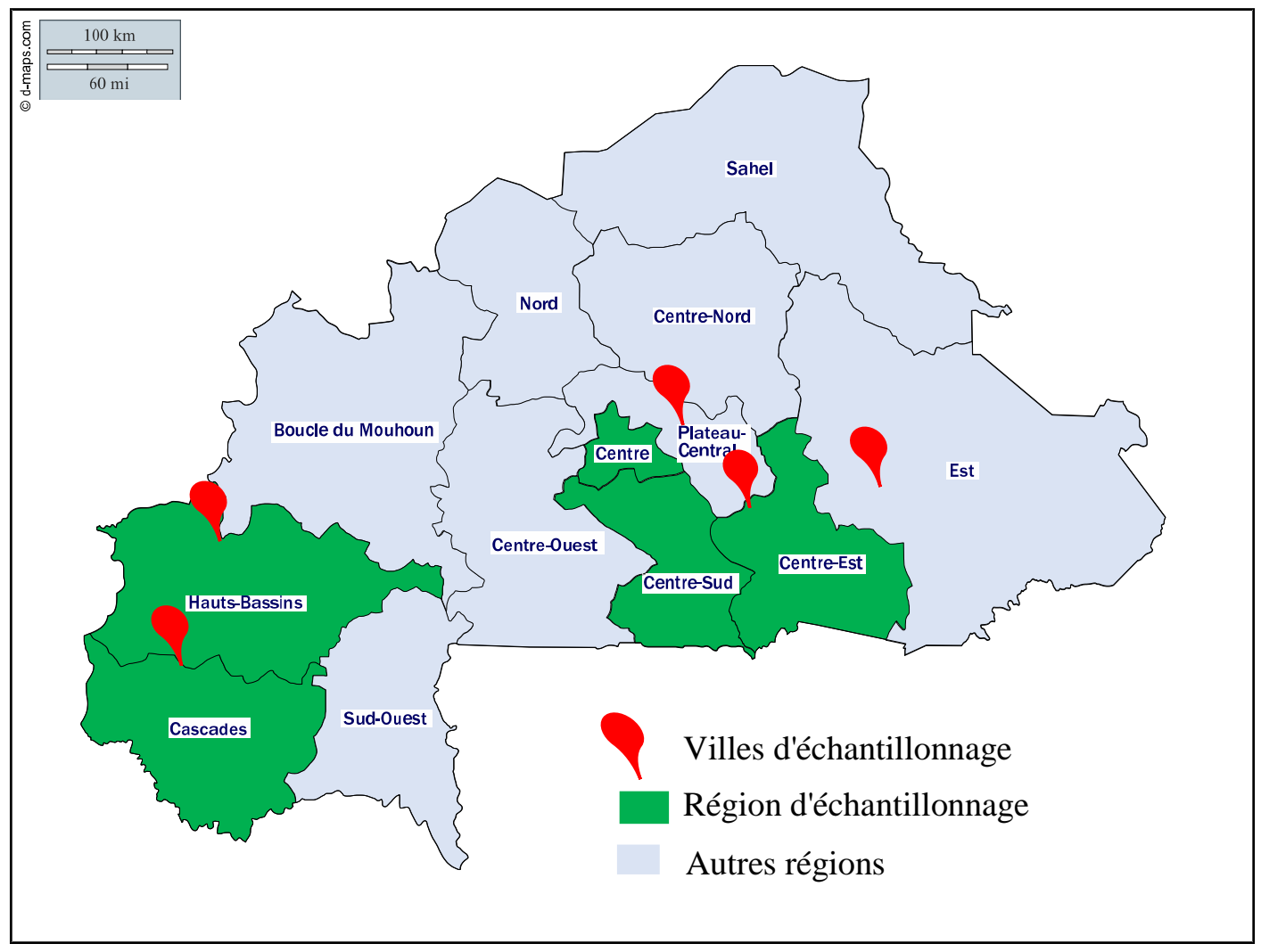

Figure 1 : Représentation des zones de l'étude sur la carte du Burkina Faso.

Tableau 1 : Valeurs des écarts à ne pas dépasser entre deux essais.

\begin{tabular}{cc}
\hline Indice de peroxyde milliéquivalents/kg & Ecarts \\
\hline Inférieur à 1 & 0,1 \\
1 à 6 & 0,2 \\
6 à 12 & 0,5 \\
Supérieur à 12 & 1 \\
\hline
\end{tabular}




\section{RÉSULTATS}

Les résultats obtenus dans cette étude ont été présentés en sept points : description des échantillons et des marques d'huile rencontrées, teneur en vitamine A des huiles, niveau de conformité de la vitamine A des échantillons d'huiles analysés, niveau de conformité de la vitamine A des huiles selon la marque, indice de peroxyde des huiles, niveau de conformité de l'indice de peroxyde par rapport aux normes en vigueur au Burkina Faso et niveau de conformité globale des huiles par rapport à leur indice de peroxyde et à leur teneur en vitamine $\mathrm{A}$.

\section{Description des échantillons collectés}

$\mathrm{Au}$ total, 59 prélèvements d'huiles réalisés dans les marchés et boutiques ont fait l'objet d'analyses physico-chimiques. Ces échantillons se répartissaient comme suit : 18 provenaient de Ouagadougou, 12 de BoboDioulasso, 10 de Cinkansé, 10 de Niangoloko et 9 de Dakola (Tableau 2). Parmi les 59 échantillons d'huile prélevés, les marques de 17 échantillons n'ont pas été précisés (HV et HVR). Les différentes marques rencontrées étaient au nombre de 11 . La répartition de ces marques par ville est représentée dans le Tableau 2.

\section{Teneur en vitamine $A$ des échantillons d'huiles analysés}

La Figure 2 montre la répartition des échantillons en fonction des villes et de la teneur en vitamine A. On constate que 40 échantillons sur 59 avaient des teneurs en vitamine A indétectables. Ceux pour lesquels les teneurs en vitamine A étaient détectables, les valeurs variaient entre $1,37 \mathrm{mg} / \mathrm{kg}$ et 21,50 $\mathrm{mg} / \mathrm{kg}$ (Figure 2). Considérant les résultats par ville, un seul échantillon sur dix était enrichi en vitamine $\mathrm{A}$ au niveau des prélèvements effectués dans la ville de Cinkansé. La moitié des échantillons étaient enrichis en vitamine A au niveau de la ville de Niangoloko. Dans les trois autres villes, les rapports étaient de 3/18 prélèvements, 4/12 et 4/9 respectivement à Ouagadougou, Bobo-Dioulasso et Dakola. Dans l'ensemble, 17/59 échantillons, soient $28,81 \%$ étaient enrichis en vitamine A.

\section{Niveau de conformité des teneurs en vitamine $A$ des échantillons d'huiles analysés}

Globalement, 45 échantillons d'huiles sur les 59 prélevés soient $76,27 \%$ étaient non conformes par rapport aux normes nationales de spécifications sur les huiles. De ces échantillons, 93,33\% avaient une teneur en vitamine $\mathrm{A}$ inférieure à la limite de détection. A Ouagadougou, la totalité des échantillons avait une teneur en vitamine A non conforme (Figure 3). Par ailleurs, à Niangoloko, la moitié des échantillons avait une teneur en vitamine A non conforme. Quant aux villes de Cinkansé, Bobo-Dioulasso et Dakola, le nombre d'échantillons non conformes sur le nombre total des échantillons prélevés dans ces villes étaient respectivement 9/10, 8/12 et 5/9.

\section{Niveau de conformité des teneurs en vitamine A des huiles selon la marque}

La Figure 4 présente le nombre de prélèvements d'huile conforme ou non conforme pour chaque marque d'huile rencontrée au cours de l'étude. La totalité des huiles de marque inconnue était non conforme par rapport à la teneur en vitamine $\mathrm{A}$. Également, les marques $H V 7$, (Huile Végétale 7), HV5, HV8 prélevées respectivement 9 fois, 5 fois et 4 fois avaient toutes des teneurs en vitamine A non conformes. Le plus grand nombre d'échantillons ayant une teneur en vitamine A conforme a été rencontré au niveau de la marque $H V 6$, avec huit prélèvements conformes sur neuf.

\section{Indice de peroxyde des huiles analysées}

La valeur moyenne des indices de peroxydes était de $5,19 \mathrm{mEq} / \mathrm{kg}$ d'huile pour l'ensemble des cinquante-neuf échantillons d'huiles avec des valeurs extrêmes de 1,37 et 
$12,36 \mathrm{mEq} / \mathrm{kg}$ (Tableau 3). Les valeurs moyennes étaient de $3,77 \mathrm{mEq} / \mathrm{kg} ; 5,02$ $\mathrm{mEq} / \mathrm{kg} ; 5,32 \mathrm{mEq} / \mathrm{kg} ; 5,34 \mathrm{mEq} / \mathrm{kg}$ et 5,82 $\mathrm{mEq} / \mathrm{kg}$ respectivement pour les villes de Dakola, Cinkansé, Bobo Dioulasso, Niangoloko et Ouagadougou.

Niveau de conformité des indices de peroxyde par rapport à la norme (CXS 2101999)

La Figure 5 montre la répartition des indices de peroxyde des huiles analysées par rapport à la valeur maximale tolérée (représentée par le trait discontinu de couleur rouge) et par rapport aux médianes des indices de peroxyde de chaque ville (représentées par des traits pleins). Pour l'ensemble des échantillons d'huile analysés, seules deux huiles sur les cinquante-neuf, soit 3,38\% avaient un indice de peroxyde non conforme à la norme CXS 210-2019.

Niveau de conformité globale des huiles par rapport à leur indice de peroxyde et à leur teneur en Vitamine A

Le nombre de non-conformité global des échantillons d'huile analysés par rapport à la norme CXS 210-2019 sur l'indice de peroxyde et à celle sur la vitamine $\mathrm{A}$ est de 46/59 échantillons, soit un taux de 77,97\%.

Tableau 2 : Répartition des échantillons d'huiles prélevées par ville et par marque.

\begin{tabular}{|c|c|c|c|c|c|c|}
\hline $\begin{array}{l}\text { Marque } \\
\text { d'huile }\end{array}$ & Ouagadougou & $\begin{array}{c}\text { Bobo } \\
\text { Dioulasso }\end{array}$ & Cinkansé & Niangoloko & Dakola & $\begin{array}{l}\text { Effectif } \\
\text { marque }\end{array}$ \\
\hline HV & 7 & 2 & - & - & - & 9 \\
\hline HV1 & - & - & - & - & 1 & 1 \\
\hline HV2 & - & 1 & - & - & - & 1 \\
\hline HV3 & 2 & - & 1 & 1 & - & 4 \\
\hline HV4 & - & - & - & & 1 & 1 \\
\hline HV5 & - & - & - & 2 & 3 & 5 \\
\hline HV6 & - & 4 & - & 5 & - & 9 \\
\hline HV7 & 5 & 4 & - & & - & 9 \\
\hline HVR & - & - & 8 & & - & 8 \\
\hline HV8 & 2 & - & - & 2 & - & 4 \\
\hline HV9 & - & 1 & - & - & - & 1 \\
\hline HV10 & 1 & - & 1 & - & 4 & 6 \\
\hline HV11 & 1 & - & - & - & - & 1 \\
\hline Effectif total & 18 & 12 & 10 & 10 & 9 & 59 \\
\hline
\end{tabular}

HV : Huile Végétale; Le numéro qui suit HV est le code attribué à la marque d'huile avant les analyses ; HVR : Huile Végétale Reconditionnée. 


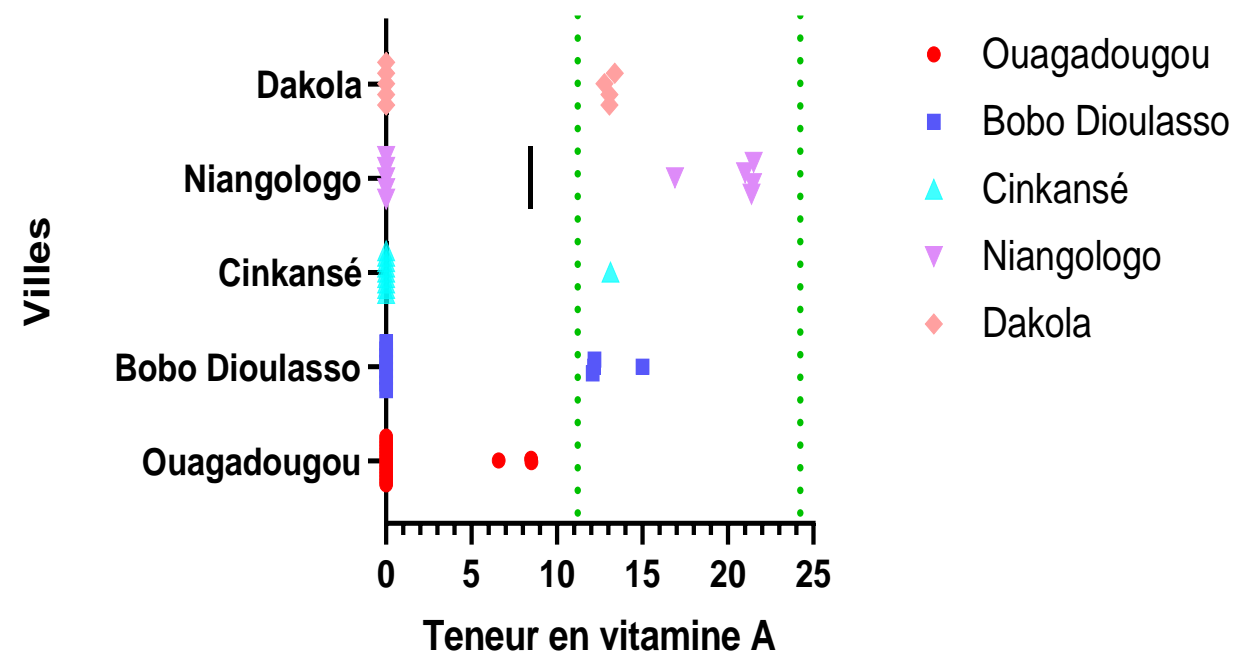

Figure 2 : Répartition des teneurs en vitamine A autour de la médiane.

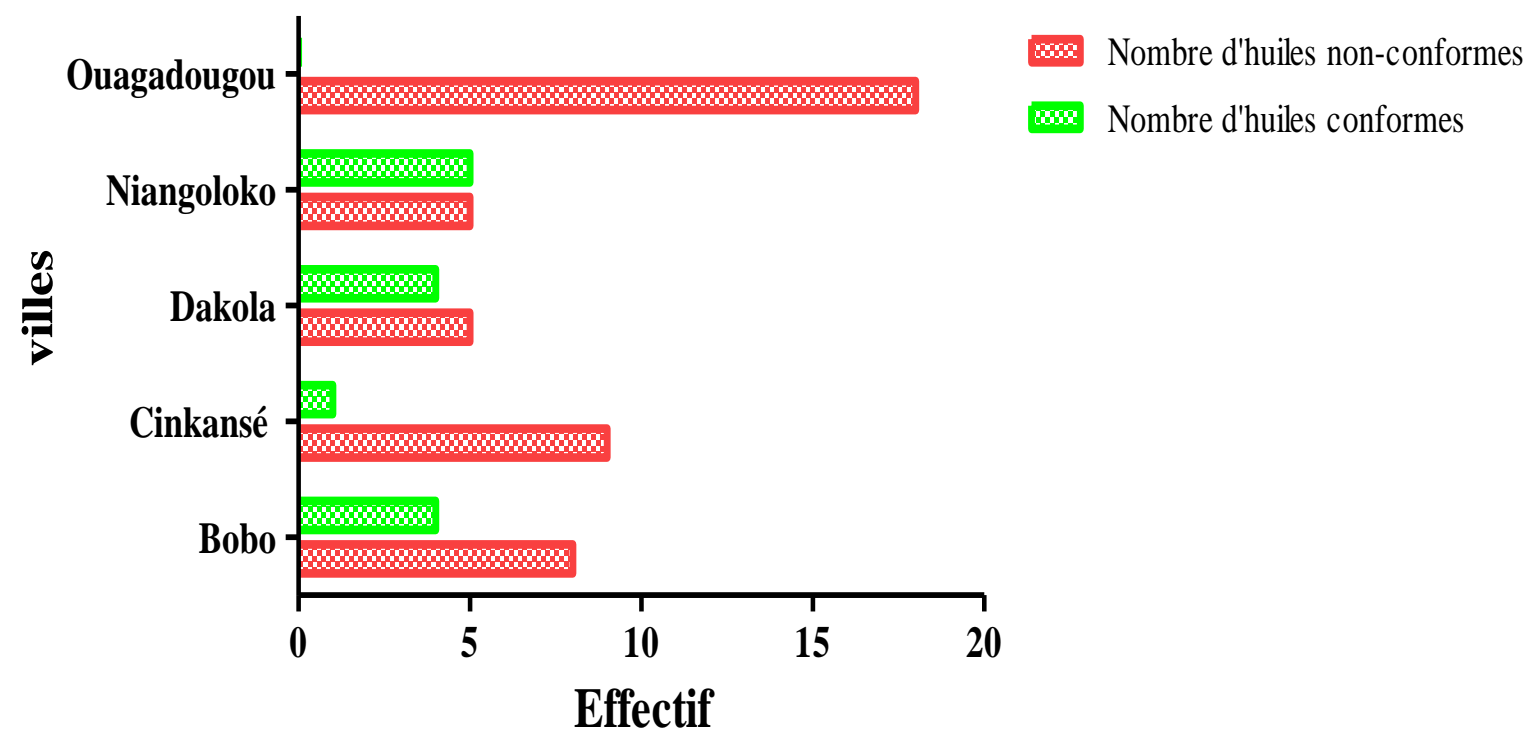

Figure 3 : Répartition des niveaux de conformité des teneurs en vitamine A des huiles selon la ville. 


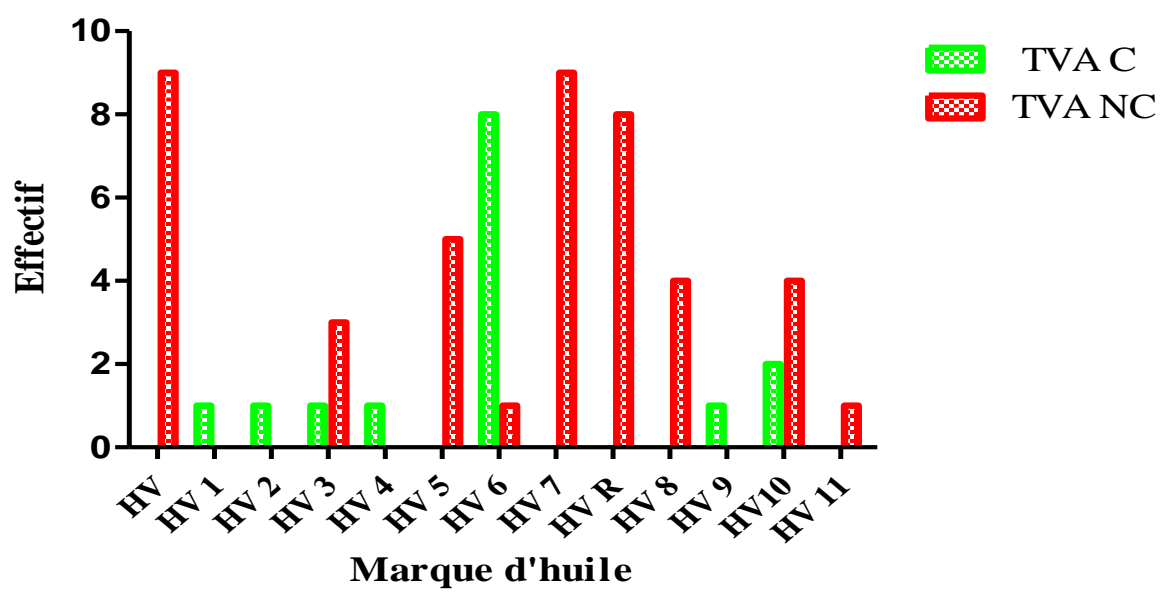

Figure 4 : Représentation des non-conformités des teneurs en vitamine A selon la marque d'huile.

TVA $C$ : Teneur en Vitamine A Conforme ;

TVA NC : Teneur en Vitamine A Non-conforme;

$H V$ : Huile Végétale ;

Le numéro qui suit HV est le code attribué à la marque d'huile avant les analyses ;

HV et HV R : sont des huiles végétales dont les marques n'ont pas été mentionnées sur les fiches par les enquêteurs.

Tableau 3 : Valeurs moyennes des indices de peroxyde des huiles analysées.

\begin{tabular}{lcccccc}
\hline Localité & Ouagadougou & $\begin{array}{c}\text { Bobo } \\
\text { Dioulasso }\end{array}$ & Cinkansé & Niangoloko & Dakola & Global \\
\hline Effectif & 18 & 12 & 10 & 10 & 09 & 59 \\
\hline $\begin{array}{l}\text { Indices de } \\
\begin{array}{l}\text { Peroxyde } \\
\text { Moyen }\end{array}\end{array}$ & 5,82 & 5,32 & 5,02 & 5,34 & 3,77 & 5,19 \\
\hline Minima & 1,37 & 2,50 & 3,62 & 2,50 & 1,37 & 1,37 \\
\hline Maxima & $\mathbf{9 , 1 2}$ & 12,36 & $\mathbf{9 , 2 4}$ & $\mathbf{8 , 4 9}$ & $\mathbf{6 , 7 4}$ & $\mathbf{1 2 , 3 6}$ \\
\hline
\end{tabular}

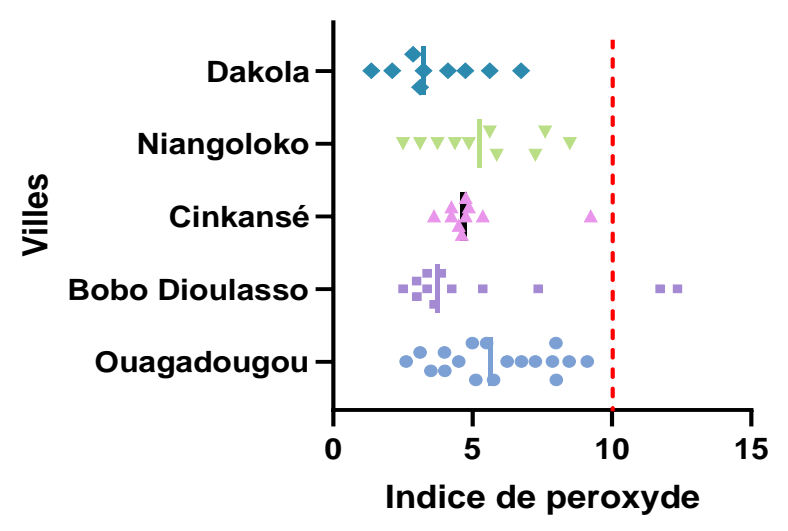

Figure 5 : Indices de peroxyde et leur niveau de conformité dans les cinq villes. 


\section{DISCUSSION}

Les huiles commercialisées au Burkina Faso proviennent d'une part de la production locale et d'autre part de l'importation. Dans le cadre de la lutte contre la carence en vitamine A, qui touche en priorité les jeunes enfants et les femmes en âge de procréer, le Burkina Faso s'est engagé dans le programme d'enrichissement en vitamine $A$, des huiles végétales depuis 2008. En 2012, le pays a adopté un arrêté interministériel $\mathrm{N}^{\circ} 2012$ 0232/MICA/MS/MEF/MAH, portant enrichissement obligatoire en vitamine A des huiles végétales produites localement ainsi que celles importées sur le territoire national. Dès lors, la conformité de la teneur en vitamine A aux normes nationales de spécifications sur les huiles raffinées est devenue une exigence de qualité nutritionnelle et sanitaire. Cependant, les résultats de cette étude révèlent un nonrespect de cet arrêté par les producteurs locaux et les importateurs des huiles végétales raffinées, car seulement, 23,73\% des échantillons analysés avaient des teneurs en vitamine A conformes. Ceci traduirait également, un non-respect des règles d'enrichissement en vitamine A de l'huile raffinée produites localement et/ou importées au Burkina Faso. Reina et al. (2017) ont trouvé $44 \%$ de conformité de la teneur en vitamine A des huiles raffinées au Cameroun.

La majeure partie des marques d'huiles rencontrées provenaient de l'importation (huit marques sur onze) La ville de Niangoloko a enregistré le plus grand nombre d'échantillons conformes (50\%) par rapport aux autres villes. Sur les 10 échantillons prélevés dans cette ville, 5 étaient de marque $H V 6$ (Huile Végétale 6), 2 étaient de marque $H V 8,1$ était de marque $H V 3$ et 2 de marque HV5. La totalité des huiles de marque $H V 6$ prélevée dans cette ville avait une teneur en vitamine A conforme. Sur l'ensemble des 59 échantillons de l'étude, cette marque (HV6) a été rencontrée 9 fois dont 8 avaient une teneur en vitamine A conforme. Cela pourrait traduire un respect des règles d'enrichissement en vitamine A dans la production de cette marque d'huile qui provient de l'importation; et un non-respect de ces règles dans la production des huiles de marque $H V 7, H V 5$ et $H V 8$ provenant toutes de l'importation. Aussi y aurait-il des insuffisances dans l'application de l'arrêté interministériel

$\mathrm{N}^{\circ} 2012-$ 0232/MICA/MS/MEF/MAH portant enrichissement obligatoire en vitamine A des huiles végétales raffinées produites et importées sur le territoire national?

La faible disponibilité des huiles adéquatement enrichies en vitamine A sur les marchés des régions de cette étude, constitue un facteur important de risque de carence en vitamine A avec toutes ses conséquences chez les personnes les plus vulnérables que sont les femmes en âge de procréer et les enfants de moins 5 ans. Le risque serait plus élevé dans la ville de Ouagadougou, car tous les échantillons d'huile qui y ont été prélevés étaient non conformes.

L'enrichissement des denrées alimentaires est un moyen très attractif et économique pour prévenir et contrôler la carence en vitamine $\mathrm{A}$, et un enrichissement alimentaire efficace pourrait suppléer la supplémentation des femmes au post-partum et des enfants plus âgés (Omar et Jose, 2002 ; Steyn et al., 2008 ; Naima et al., 2019).

Les peroxydes sont des intermédiaires dans la réaction d'oxydation conduisant à la détérioration des lipides. Par conséquent, un indice de peroxyde élevé est une indication de la sensibilité d'une huile au rancissement (Belewu et al., 2010 ; Akinterinwa et al., 2015). L'indice de peroxyde permet d'apprécier les premières étapes d'une détérioration oxydative de l'huile (Cuvelier et Maillard, 2007 ; 2012). Dans cette étude, seuls deux échantillons avaient des indices de peroxyde dépassant la limite maximale tolérée. Ces échantillons ont été rencontrés dans la ville de Bobo-Dioulasso. Ces indices élevés pourraient s'expliquer soient par la composition de ces huiles, soient 
par la durée ou les conditions de stockage. Selon le profil en acides gras (proportion d'Acides Gras Insaturées) et en composants mineurs (teneur en vitamine E), la stabilité à l'oxydation des huiles varie fortement. Une forte concentration en Acides Gras PolyInsaturés (AGPI) rendrait l'huile végétale plus sujette à l'oxydation pendant le stockage. Cette oxydation se produirait même plus vite si l'huile est exposée aux rayons ultraviolets, à la chaleur ou aux ions métalliques comme le $\mathrm{Fe}^{2+}$ ou le $\mathrm{Cu}^{2+}$ (Dubois et al., 2007). Selon la durée du stockage, Andarwulan et al. (2014) ont trouvé que lorsque l'indice de peroxyde de l'huile était inférieur à $2 \mathrm{mEq} \mathrm{O}_{2} / \mathrm{kg}$, cette huile avait une durée de conservation comprise entre 2 et 3 mois, lorsqu'il était d'environ $4 \mathrm{mEq}$ $\mathrm{O}_{2} / \mathrm{kg}$, la durée de conservation était de quelques semaines et celle de l'huile d'environ $9 \mathrm{mEq} \mathrm{O} / 2 / \mathrm{kg}$, de quelques jours seulement. La courte durée de conservation d'une huile de cuisson de $9 \mathrm{mEq} \mathrm{O}_{2} / \mathrm{kg}$, pourrait avoir un impact négatif sur la santé, en raison de l'augmentation potentielle des radicaux libres de la peroxydation lipidique dans l'huile. Dans cette étude, 39 échantillons avaient un indice de peroxyde supérieur à $4 \mathrm{mEq} \mathrm{O}_{2} / \mathrm{kg}, 25$ avaient un indice de peroxyde supérieur à 5 $\mathrm{mEq} \mathrm{O}_{2} / \mathrm{kg}$ et 4 échantillons avaient un indice de peroxyde supérieur à $9 \mathrm{mEq} \mathrm{O}_{2} / \mathrm{kg}$. Selon les résultats de Andarwulan et al. (2014), les 4 huiles ayant $9 \mathrm{mEq} \mathrm{O}_{2} / \mathrm{kg}$ ne devraient même plus être conservées au risque d'exposer la santé des consommateurs. Almeck et al. (2008) ont observé, une augmentation significative de l'indice de peroxyde de l'huile de la pulpe des fruits de Canarium schweinfurthii Engl., pendant un temps de stockage de 10 mois à $25^{\circ} \mathrm{C}$. Selon les conditions de stockage, Faustine et al. (2016), ont trouvé que les huiles stockées dans l'obscurité avaient un indice de peroxyde faible, comparé à celles qui étaient stockées à l'air libre pendant une période de 60 jours. De ce fait, la conservation des huiles à l'obscurité permettrait de ralentir leur dégradation par oxydation.
Arnaud et al. (2012) ont observé une augmentation de la décomposition de la vitamine A dans l'huile végétale enrichie, une fois que les niveaux de peroxyde y étaient supérieurs à $2 \mathrm{mEq} \mathrm{O}_{2} / \mathrm{kg}$. Parmi les indices de peroxyde des huiles prélevées au cours de cette étude, seule une huile avait une valeur de peroxyde inférieure à $2 \mathrm{mÉq} \mathrm{O}_{2} / \mathrm{kg}$. Ce qui permet de prédire une décomposition probable de la vitamine A contenue dans les 58 autres échantillons. Cela implique qu'une attention particulière soit accordée à la valeur de l'indice de peroxyde par les producteurs avant l'étape d'enrichissement en vitamine A de l'huile.

\section{Conclusion}

Cette étude avait pour but d'évaluer l'application de la politique nationale portant enrichissement obligatoire des huiles végétales raffinées en vitamine A au Burkina Faso. Elle a permis de relever que seulement $28,81 \%$ des huiles couramment consommées au Burkina Faso étaient enrichies en vitamine A. Les huiles adéquatement enrichies en vitamine A ne représentaient que $23,73 \%$ des huiles analysées. Ce qui constitue un problème quant au respect de l'arrêté interministériel №20120232/MICA/MS/MEF/MAH sur la production et l'importation des huiles végétales raffinées au Burkina Faso. Cela aura pour conséquence évidente la non-atteinte de l'objectif visé par l'enrichissement des huiles végétales raffinées en vitamine $\mathrm{A}$, à savoir la réduction de la carence en vitamine A au Burkina Faso, notamment chez les couches les plus vulnérables que sont les femmes en âge de procréer et les enfants de moins de 5 ans. L'indice de peroxyde des huiles analysées était acceptable pour la majorité des échantillons. Seulement deux échantillons avaient un indice de peroxyde non conforme par rapport à la valeur limite fixée par le codex Alimentaruis. Cependant, la conservation des huiles ayant un indice de peroxyde supérieur à $4 \mathrm{mEq} \mathrm{O}_{2} / \mathrm{kg}$ représenterait un danger pour la santé des consommateurs. Le suivi de l'indice de 
peroxyde des huiles conformes sur une période de 6 mois s'impose alors pour évaluer leur stabilité sur le long terme.

\section{REMERCIEMENTS}

$\mathrm{Au}$ terme de ce travail, nous tenons à remercier :

- L'institut national de santé publique (INSP) pour la collaboration scientifique ;

- Le Comité d'éthique de la recherche en santé pour avoir autorisé la réalisation de cette étude; - Les directions régionales de la santé des régions du Centre, des Hauts-Bassins, des Cascades et du Centre-Est pour avoir autorisé la réalisation de cette étude ;

- Les populations des régions du Centre, des Hauts-Bassins, des Cascades et du Centre-Est pour leur collaboration.

\section{CONFLIT D'INTERETS}

Les auteurs déclarent qu'ils n'ont pas d'intérêts concurrents.

\section{CONTRIBUTIONS DES AUTEURS}

EK est l'investigateur principal de l'étude. TMOK a conduit les analyses de laboratoire et rédigé le manuscrit. FN a facilité la conduite des analyses au laboratoire. DSK a contribué dans la rédaction du manuscrit et à sa révision. MKAC a contribué dans la rédaction du manuscrit et à sa révision. BSRB a contribué dans la rédaction du manuscrit et à sa révision. ABS a contribué à la collecte des échantillons d'huile et à leur traitement au laboratoire. OUS a participé à la réalisation des analyses et au traitement des données. SS a participé à la réalisation des analyses et au traitement des données. II a participé à la réalisation des analyses et au traitement des données. NSDM a participé à la rédaction du manuscrit. NRM a contribué à la relecture du manuscrit. BPOS a participé à la relecture du manuscrit. HH est l'initiateur du projet de recherche objet de cet article. MB a facilité la rédaction du manuscrit, a participé à sa validation et à sa relecture. AY a supervisé la collecte des échantillons et a contribué à la correction du manuscrit. CSK a coordonné les activités de prélèvement. $\mathrm{HK}$ a facilité la réalisation de l'étude à travers une gestion efficiente des fonds alloués.

\section{RÉFÉRENCES}

Almeck K, Aboubakar D, Clergé T, Michel P. 2008. Evolution de quelques paramètres de qualité physico-chimique de l'huile de la pulpe des fruits de Canarium schweinfurthii Engl. au cours du stockage. International Journal of Biological and Chemical Sciences; 2(3): 249-257. DOI: 10.4314/ijbcs.v2i3.39760

Andarwulan, N, Gitapratiwi D, Laillou A, Fitriani D, Hariyadi P, Moench-Pfanner R, Martianto D. 2014. Quality of vegetable oil prior to fortification is an important criteria to achieve a health impact. Nutrients, 6: 5051-5060. DOI: https://doi.org/10.3390/nu6115051

Arnaud L, Saeb AH, Amal HM, Mohamed M, Fabian R, Sonia F, Jacques B, Nabih AI, Regina M. 2012. Vegetable oil of poor quality is limiting the success of fortification with vitamin A in Egypt. Food and Nutrition Bulletin, 33(3): 186-193.

DOI: 10.1177/156482651203300303

Belewu MA, Adekola FA, Adebayo GB, Ameen OM, Muhammed NO, Olaniyan AM, Adekola OF, Musa AK. 2010. Physico-chemical characteristics of oil and biodiesel from Nigerian and Indian Jatropha curcas seeds. International Journal of Biological and Chemical Sciences, 4(2): 524-529. DOI: 10.4314/ijbcs.v4i2.58169

Naima A, Asmaa EL H, Amina B, Khalid E, Abdeslam H, Mohammed EL M, Marjorie JH, Najat M, Hassan A. 2019. Combined consumption of a single high-dose vitamin A supplement with provision of vitamin A fortified oil to households maintains adequate milk 
retinol concentrations for 6 months in lactating Moroccan women. Applied Physiology, Nutrition and Metabolism, 45(3):275-282. DOI: 10.1139/apnm2019-0116

Citelli M, Bittencourt LL, Da Silva SV, Pierucci AP, Pedrosa C. 2012. Vitamin A modulates the expression of genes involved in iron bioavailability. Biological Trace Elements Research, 149: 64-70. DOI: https://doi.org/10.1007/s12011-0129397-6

Cusick SE, Tielsch JM, Ramsan M, Jape JK, Sazawal S, Black RE, Stoltzfus RJ. 2005. Short-term effects of vitamin A and antimalarial treatment on erythropoiesis in severely anemic Zanzibari preschool children. American Journal of Clinical Nutrition, 82: 406412. DOI:10.1093/ajen/82.2.406

Cuvelier ME, Maillard MN. 2007. Comment évaluer l'efficacité des antioxydants alimentaires? Science des Aliments, 27: 259-82. DOI: 10.3166/sda.27.259-282

Cuvelier ME, Maillard MN. 2012. Stabilité des huiles alimentaires au cours de leur stockage. Oléagineux, Corps Gras, Lipides, 19(2): 125-132. DOI: 10.1051/ocl.2012.0440

Dubois V, Breton S, Linder M, Fanni J, Parmentier M. 2007. Fatty acid profiles of 80 vegetable oils with regard to their nutritional potential. European Journal of Lipid Science and Technology, 109(7): 710-732. DOI: 10.1002/ejlt.200700040

FAO/WHO. 2015. Second International Conference on nutrition Report. FAO/WHO, Rome, 17 p.

Faustine NN, Tupeligwe RM, Stephen SN. 2016. Stability of traditionally processed vegetable oils and their blends under different storage conditions International Journal of Biological and Chemical Sciences,
10(5): $\quad 2341-2350 . \quad$ DOI: 10.4314/ijbcs.v10i5.31

Hoag KA, Nashold FE, Goverman J, Hayes CE. 2002. Retinoic acid enhances the T helper 2 cell development that is essential for robust antibody responses through its action on antigen presenting cells. Journal of Nutrition, 132: 37363739.

DOI: https://doi.org/10.1093/jn/132.12.3736

Barreto da Cunha MDS, Hankins NAC, Arruda SF. 2018. Effect of vitamin A supplementation on iron status in humans: a systematic review and metaanalysis. Critical Reviews in Food Science and Nutrition, 2018: 1-15. DOI: 10.1080/10408398.2018.1427552

Ministère de la Santé. 2012. Arrêté interministériel $\quad \mathrm{N}^{\circ} 2012$ 0232/MICA/MS/MEF/MAH portant enrichissement obligatoire des huiles végétales raffinées en vitamine $A$ et de la farine de blé tendre en fer et en acide folique. Ministère de la Santé, Burkina Faso.

Ministère de la Santé. 2020. Plan stratégique multisectoriel de nutrition 2020-2021. Ministère de la Santé, Burkina Faso, 45p.

Ministère du Commerce, de l'Industrie et de l'Artisanat. 2020. Communiqué N 020/0014 /MICA/SG/DGU-CI. Ministère du Commerce, de l'Industrie et de l'Artisanat, Burkina Faso.

Bendech MA, Acakpo A, Aguayo V, Baker S, Diene SM, Lathen L, Ouedraogo A. 2000. Les pratiques prometteuses et les leçons apprises dans la lutte contre la carence en vitamine A dans les pays de l'Afrique subsaharienne. Projet BASICS de l'USAID, USA, 67p.

Omar D, Jose OM. 2002. Food fortification to reduce vitamin a deficiency: international vitamin a consultative group recommendations. Journal of 
Nutrition, 132(9): 2927S-2933S. DOI: https://doi.org/10.1093/jn/132.9.2927S

Organisation Mondiale de la Santé. 2011.

Directives Sur L'enrichissement Des Aliments En Micronutriments. OMS, Rome, 379 p.

Reina E-S, Martin N, Alex N, MarieMadeleine G, Avital F, Marjorie JH, Ann T, Kenneth HB. 2017. Vitamin A Status of Women and Children in Yaoundé and Douala, Cameroon, is Unchanged One Year after Initiation of a National Vitamin A Oil Fortification Program. Nutrients, 9(5): 522. DOI: 10.3390/nu9050522

Schroeder SE, Reddy MB, Schalinske KL. 2007. Retinoic acid modulates hepatic iron homeostasis in rats by attenuating the RNA-binding activity of iron regulatory proteins. Journal of
Nutrition, $\quad$ 137(12): $\quad$ 2686-2690. DOI:10.1093/jn/137.12.2686

Steyn NP, Nel J, Labadarios D. 2008. Will fortification of staple foods make a difference to the dietary intake of South African children? Sud African Journal of Clinical Nutrition, 21(1): 22-26.

Tao L. 2015. Oxidation of polyunsaturated fatty acids and its impact on food quality and human health. Advance Food Technology Nutrition Sciences Open Journal, 1(6): 135-142. DOI: 10.17140/AFTNSOJ-1-123

Youna MH, Laura F, Regina M-P, Arnaud L, Jacques B, Cécile R, Sylvie A. 2015. Influence of light exposure and oxidative status on the stability of vitamins A and D3 during the storage of fortified soybean oil. Food Chemistry, 184: $90-98 . \quad$ DOI: 10.1016/j.foodchem.2015.03.096 